REVISTA MATEMATICA de la

Universidad Complutense de Madrid

Volumen 5, nümero ]; 1992.

http://dx.doi.org/10.5209/rev_REMA.1992.v5.n1.17936

\title{
Conos de Luz
}

\author{
JAVIER LAFUENTE LÓPEZ
}

ABSTRACT. Using a new characterization of the Lorentz quadrics, we establish a reformulation of the Special Relativity Theory.

\section{RESUMEN}

Un punto central de una figura $F$ de un espacio proyectivo $\mathbf{E}$, es un punto $c$, que es centro de simetría de $F$ respecto a alguna restricción afín de $\mathbf{E}$, y cada recta que pasa por $c$ corta a $F$ en exactamente dos puntos.

En la primera parte del trabajo, se caracteriza a las cuádricas de Lorentz, como las únicas figuras cerradas del espacio proyectivo cuyo conjunto de puntos centrales tiene interior no vacío.

En la segunda parte, se hace una breve incursión a los modelos geométricos de la cinemática clásica de partículas, para mostrar cómo el resultado anterior permite reformular el Principio Fundamental de la Relatividad Especial, y obtener el espacio de Minkowski como modelo de la cinemática relativista.

\section{INTRODUCCION}

Se denomina punto interior de una cuádrica $\mu$ de un espacio proyectivo real $\mathbf{E}$ (de dimensión $n \geq 2$ ) a un punto $p \in \mathbf{E}$ desde el cual no puede trazarse una recta tangente a $\mu$. El punto $p$ se denomina exterior a $\mu$ si no es interior,

1991 Mathemmatics Subject Classification: 83A05, 51B20.

Editorial Complutense. Madrid, 1992. 
ni pertenece a la cuádrica. Denotamos por im $\mu$ al conjunto de puntos de la cuádrica, y por int $\mu$ (ext $\mu$ ) al conjunto de puntos interiores (exteriores).

Los conjuntos int $\mu$, im $\mu, \mathrm{y}$ ext $\mu$ inducen una partición del conjunto de puntos de $\mathbf{E}$ que se corresponde con la clasificación subordinada por el grupo $\mathrm{GP}(\mathbf{E}, \mu)$ de transformaciones proyectivas que dejan invariante la cuádrica, al actuar sobre los puntos de $\mathbf{E}$.

Se prueba que si $\mu$ es una cuádrica de $\mathbf{E}$ con puntos interiores (es decir, int $\mu \neq \varnothing)$ entonces respecto a un sistema de coordenadas homogéneas [ $\left.x_{1}\right]$ adecuadamente elegido, las ecuaciones de $\mu$ son de la forma:

$$
\varepsilon x_{0}^{2}+\sum_{l=1}^{n} x_{l}^{2}=0 \quad(\varepsilon= \pm 1)
$$

Cuando $\varepsilon=1$, la cuádrica se llama euclídea, y el conjunto de puntos interiores, int $\mu$ coincide con $\mathbf{E}$, mientras que im $\mu=$ ext $\mu=\varnothing$.

Cuando $\varepsilon=-1$, la cuádrica se llama de Lorentz. Una cuádrica $\mu$ de Lorentz puede, por tanto, caracterizarse por las condiciones int $\mu \neq \varnothing$, y ext $\mu \neq \varnothing$ (que equivalen a int $\mu \neq \varnothing, \mathrm{e}$ im $\mu \neq \varnothing$ ). Se tiene el siguiente resultado.

Lema 1.1 ([4] vol 4 pág 73).

Si $\mu$ es una cuádrica de Lorentz y $p \in \mathbf{E}$, entonces:

El punto p es interior a $\mu$ si y sólo si, cada recta proyectiva $\Delta$ que pasa por $p$, corta al conjunto de puntos de la cuádrica, im $\mu$ en exactamente dos puntos distintos $a, b$.

Por oira parte, si $p^{\perp}$ denota al hiperplano polar, de p "respecto a $\mu$, entonces $p, \Delta \cap p^{\perp}, a, b$ son puntos armónicamente separados, es decir: $\left[p, \Delta \cap p^{\downarrow} ; a, b\right]=-1$

En particular se tiene que la razón simple $(p: a: b)$ en la recta afín $\Delta-\Delta \cap p^{\perp}$ es igual a $-1, y$, por tanto, $p$ es centro de simetría de im $\mu$ en el

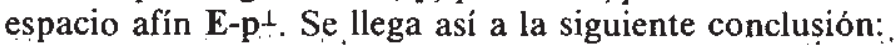

\section{Proposición 1.2}

Si $\mu$ es una cuádrica de Lorentz, entonces el conjunto $\mathscr{C}=i m, \mu$ es cerrado, y verifica la siguiente propiedad para sodo $p \in$ int $\mu$ : 
(1) Cada recta proyectiva $\Delta$ que pasa por $p$ corta a $\mathscr{C}$ en exactamente dos puntos.

(2) Existe $H$ hiperplano de $\mathbf{E}$ tal que p es centro de simetria de $\mathscr{C}$ en el espacio afin $\mathbf{E}-H$.

El conjunto $\mathscr{C}$ es entonces un cono de luz, según la siguiente definición:

Definición 1.3: Cono de luz

Sea $\mathscr{C}$ un subconjunto no vacio de un espacio proyectivo $\mathbf{E}$.

a) Un punto $p \in E$, se llama punto central de $\mathscr{L}$, si:

1) Cada recta que pasa por $p$ corta a $\mathscr{L}$ en exactamente dos puntos distintos.

2) Existe un hiperplano $H$ de $\mathbf{E}$ de forma que $p$ es centro de simetria de $\mathscr{C}$ en el espacio afín $\mathbf{E}-H$. Se denomina a $H$ hiperplano central de $p$.

b) Se dice que $\mathscr{C}$ es un cono de luz, si es cerrado, y el conjunto $\mathscr{D}$ de sus puntos centrales tiene interior no vacio.

El propósito de este artículo es demostrar el siguiente

\section{Teorema del cono}

Si $\mathscr{C}$ es un cono de luz en el espacio proyectivo real $\mathbf{E}$, entonces existe una (única) cuádrica de Lorentz $\mu$ en $\mathbf{E}$ tal que:

$$
\operatorname{im} \mu=\mathscr{b}
$$

El esquema para la demostración del teorema del cono es el siguiente:

Sea p un punto interior a $\mathscr{D}$ y sea $D$ un abierto conexo con $p \in D \subseteq \mathscr{D}$. Se probará entonces la aplicación:

$$
D \ni a \rightarrow \mathbf{H}(a) \in \mathbf{E}^{*}
$$

que hace corresponder a cada punto $a \in D$ su hiperplano central $\mathbf{H}(a)$, preserva la razón doble, e induce una única polaridad:

$$
\mathbf{E} \ni a \rightarrow \overline{\mathbf{H}}(a) \in E^{*}
$$


con $\overline{\mathbf{H}} \mid D=\mathbf{H}$. Esta polaridad define una cuádrica $\mu$ que tiene a $\mathscr{D}$ por conjunto de puntos interiores (es, por tanto, cuádrica de Lorentz) e im $\mu=\mathscr{C}$.

Se requieren algunos preparativos:

\section{APLICACIONES QUE PRESERVAN LA RAZON DOBLE}

En este epígrafe $\mathbf{E}$ y $\mathbf{E}^{\prime}$ denotarán espacios proyectivos reales de la misma dimensión $n \geq 2$. Si $S$ es un sistema de puntos, se denotará por $\langle S\rangle$ el subespacio proyectivo generado.

Comenzaremos estableciendo algunas definiciones preliminares:

\section{Definición 2.1}

a) Un conjunto $D$ de $\mathbf{E}$ se llama convexo, si para todo par de puntos a, $b \in D(a \neq b)$, alguna de las dos componentes conexas del conjunto $\langle a, b\rangle-\{a, b\}$ está contenida en $D$. El conjunto $[a, b]_{D}$ denota una componente de $\langle a, b\rangle-\cdots a, b\}$ contenida en D. (Si ambas componentes están contenidas en $D$ entonces $[a, b]_{D}$ denota a cualquiera de ellas.)

b) Dados cuatro puntos $a, b, c, d$ de $\mathbf{E}$, la razón doble $[a, b ; c, d]$ está definida, si los cuatro puntos están alineados, y al menos hay tres distintos.

El convenio que seguiremos para definir la razón doble es el siguiente:

Si $a, b, c$ son tres puntos distintos de una recta proyectiva real $\Delta$, existe una única homografía $h: \Delta \rightarrow \widetilde{\mathbf{R}}=\mathbf{R} \cup\{\infty\}$ con $h(a)=\infty, h(c)=1$. Entonces:

$$
[a, b ; x, c]=h^{\prime \prime}(x) \text { para todo } x \in \Delta
$$

En particular: $[a, b ; a, c]=0,[a, b ; b, c]=\infty,[a, b ; c, c]=1$.

Nótese que si $\Delta=\tilde{\mathbf{R}}$, es $[a, \infty ; x, c]=(a: x: c)=\frac{x-a}{c-a}, y[a, b ; x, c]=\frac{(a: x: c)}{(b: x: c)}$ cuando $a, b, x, c$ son puntos de $\mathbf{R}$ y además $x \neq b$.

Las igualdades $[a, b ; x, c]=[a, b ; c, x]^{-1}=[b, a ; x, c]^{-1}=[x, c ; a, b]$ permiten extender el concepto de razón doble $[a, b ; x, c]$ cuando en $\{a, b, x, c]$ hay al menos tres puntos distintos.

\section{Observación 2.2}

No es difícil probar que si $D$ es convexo y el subespacio $\langle D\rangle$ generado por $D$ coincide con el espacio total $\mathbf{E}$, entonces int $D \neq \phi$. 


\section{Definición 2.3}

Sea $D$ un subconjunto de un espacio proyectivo E. Una aplicación $f: \mathrm{D} \rightarrow \mathbf{E}^{\prime}$ se dice que preserva la razón doble, cuando para cada cuaterna $a$, $b, c, d$ de puntos de $D$ para los que $[a, b ; c, d]$ está definida, se verifica que $[f(a), f(b) ; f(c), f(d)]$ esiá definida, y además:

$$
[a, b ; c, d]=[f(a), f(b) ; f(c), f(d)]
$$

El objetivo es probar que toda aplicación $f: D \rightarrow \mathbf{E}^{\prime}$ con dominio $D$ un abierto conexo de $\mathbf{E}$, si preserva la razón doble, es restricción de una única homografía $\bar{f}: \mathbf{E} \rightarrow \mathbf{E}^{\prime}$.

\section{Proposición 2.4}

Sea $D$ un subconjunto convexo de $\mathbf{E}, y f: D \rightarrow \mathbf{E}^{\prime}$ una aplicación que preserva la razón doble. Entonces para $a, b, c \in D$ se verifica la equivalencia:

$$
c \in\langle a, b\rangle \Leftrightarrow f(c) \in\langle f(a), f(b)\rangle
$$

\section{Demostración:}

La aplicación $f$ es claramente inyectiva, pues si $a, b \in D, a \neq b, y f(a)=f(b)$, entonces puden tomarse $d, e \in(D \cap<a, b>), d \neq e$, y por tanto:

$$
[a, d ; b, e] \neq 0=[f(a), f(d) ; f(b), f(e)] \text {. Así } f(a) \neq f(b) .
$$

Veamos que si $a, b, c$ son puntos de $D$ tales que $a^{\prime}=f(a), b^{\prime}=f(b), c^{\prime}=f(c)$, están alineados entonces necesariamente también lo están $a, b$, y $c$.

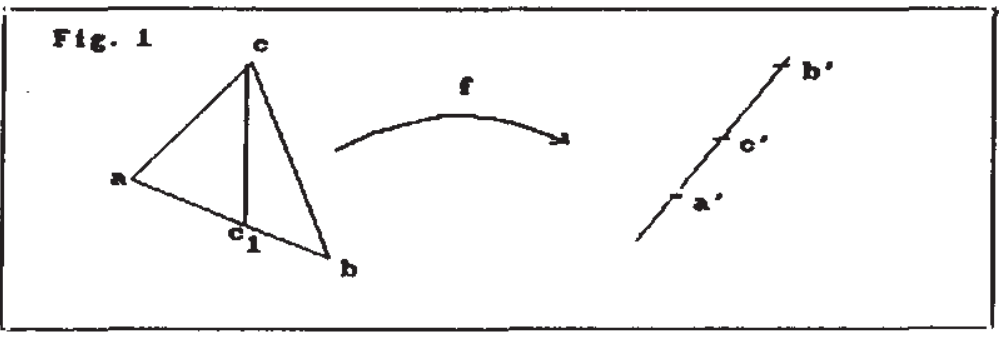

En efecto, supuesto que $a, b$, y $c$ no están alineados se ve que la imagen de $[a, c]_{D} \cup[c, b]_{D} \cup[b, a]_{D}$ coincide necesariamente con la recta $\Delta^{\prime}$ que contiene a los puntos $a^{\prime}, b^{\prime}, c^{\prime}$. Por otra parte, tomando $c_{1} \in[a, b]_{D}-\{a, b\}$, el 
segmento $\left[c, c_{1}\right]_{D}$ se aplica también sobre $\Delta^{\prime}$, lo que contradice la inyectividad de $f$.

\section{Corólario 2.5}

Si $D$ es convexo de $\mathbf{E}, y$ f: $D \rightarrow \mathbf{E}^{\prime}$ preserva la razón doble, entonces $f(D)$ es convexo de $\mathbf{E}^{\prime}, y f^{-1}: f(D) \rightarrow \mathbf{E}$ preserva la razón doble.

\section{Proposición 2.6}

Sea $D$ un subconjunto convexo de $\mathbf{E}, y \quad f: D \rightarrow \mathbf{E}^{\prime}$ una aplicación que preserva la razón doble. Entonces para $a, a_{0}, \ldots, a_{k} \in D$ se verifica la equivalencia:

$$
\text { ... } \quad a \in<a_{0}, \ldots, a_{k}>\Leftrightarrow f(a) \in<f\left(a_{0}\right), \ldots f\left(a_{k}\right)>
$$

\section{Demostración:}

Por el anterior corolario, es suficiente probar la implicación

$$
. a \in<a_{0}, \ldots, a_{k}>\Rightarrow f(a) \in<f\left(a_{0}\right), \ldots f\left(a_{k}\right)>
$$

ya que esta támbién sería válida para $f^{-1}: f(D) \rightarrow \mathbf{E}$.

La implicación se verifica para $k=1$, por el corolario 2.5. Probémoslo por inducción para $k \geq 2$ :

Podemos suponer de entrada, que $\left(a_{0}, \ldots, a_{k}\right)$ es proyectivamente independiente, $a \in\left\langle a_{0}, \ldots, a_{k}\right\rangle \cap D=D_{k}$, y que $a \notin\left\langle a_{1}, \ldots, a_{k}\right\rangle$. Sea $b$ un punto de $\left\langle a_{1}, \ldots, a_{k}\right\rangle \cap D$ interior a $D_{k}$ (que existe por 1.3).

Si $a \in<a_{0}, b>$ por la hipótesis de inducción:

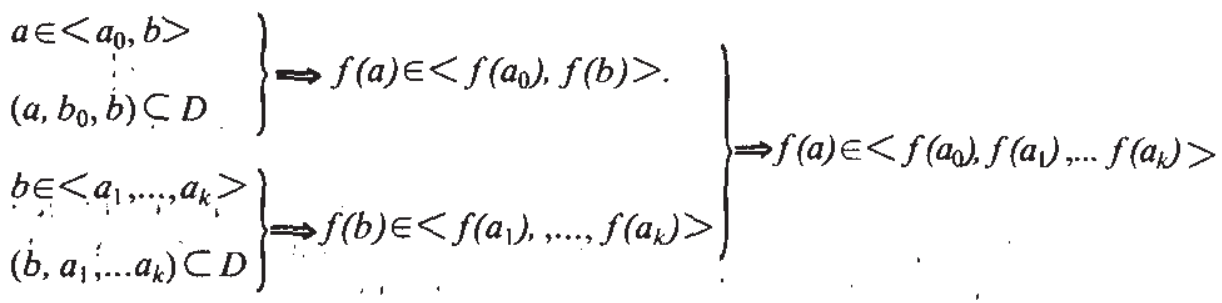




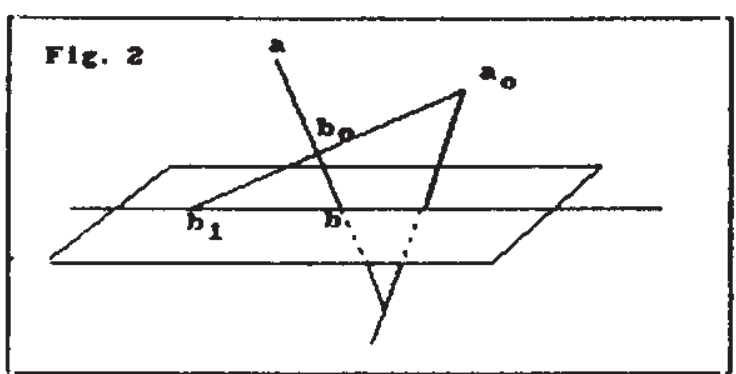

Si $a \notin<a_{0}, b>$ entonces $P=\left\langle a, a_{0}, b>\right.$ es un plano que corta a $\left\langle a_{1}, \ldots, a_{k}\right\rangle$ en una recta $\Delta$ que contiene al punto $b$, y existe un punto $b_{0}$ en alguna componente conexa de $\left(\langle a, b>-\{a, b\}) \cap D\right.$ tal que $b_{1}=\left\langle a_{0}, b_{0}\right\rangle \cap \Delta \in D$.

Nuevamente por la hipótesis de inducción se tiene:

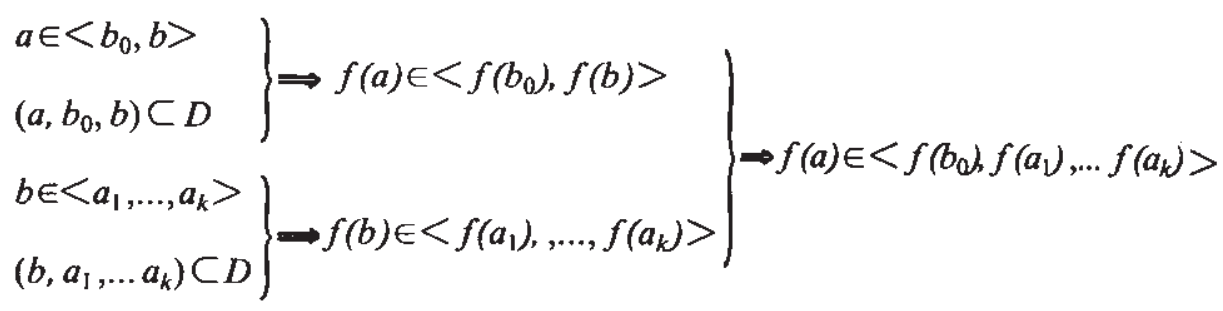

Veamos por último que $<f\left(b_{0}\right), f\left(a_{1}\right), \ldots, f\left(a_{k}\right)>=<f\left(a_{0}\right), f\left(a_{1}\right), \ldots$, $f\left(a_{k}\right)>$.

En efecto, como $a_{0} \in\left\langle b_{0}, b_{1}\right\rangle$, y $b_{1} \in\left\langle a_{1}, \ldots, a_{k}\right\rangle$, por la hipótesis de inducción es, $\left.f\left(a_{0}\right) \in<f\left(b_{0}\right), f\left(b_{1}\right)\right\rangle$ y $f\left(b_{1}\right) \in\left\langle f\left(a_{1}\right), \ldots, f\left(a_{k}\right)>\right.$ luego:

$$
f\left(a_{0}\right) \in<f\left(b_{0}\right), f\left(a_{1}\right), \ldots f\left(a_{k}\right)>
$$

Análogamente se ve que como $b_{0} \in\left\langle a_{0}, b_{1}\right\rangle$ y $b_{1} \in\left\langle a_{1}, \ldots, a_{k}\right\rangle$, es:

$$
f\left(b_{0}\right) \in<f\left(a_{0}\right), f\left(a_{1}\right), \ldots f\left(a_{k}\right)>
$$

\section{Teorema 2.7}

Sea $D$ un abierto conexo de $\mathbf{E}$. Si $f: D \rightarrow \mathbf{E}^{\prime}$ es una aplicación que preserva la razón doble, entonces existe una única homografia $\bar{f}: \mathbf{E} \rightarrow \mathbf{E}^{\prime}$, que extiende a $f($ es decir $\bar{f} \mid D=f)$. 


\section{Demostración:}

Probaremos en primer lugar, que para cada $e_{0} \in D$ existe $U$ entorno de $e_{0}$ contenido en $D$, y $f_{U}: \mathbf{E} \rightarrow \mathbf{E}^{\prime}$ homogratia tal que $f_{U}=f$ en $U$.

Sea $\varepsilon=\left(e_{0}, e_{1}, \ldots, e_{n} ; u\right) \subset D$ un sistema de referencia proyectivo de $\mathbf{E}$. Denotamos por $H_{1}=\left\langle e_{0}, \ldots, \hat{e}_{i}, \ldots, e_{n}\right\rangle$ donde $\hat{e}_{i}$ significa que el punto $e_{i}$ no aparece en el sistema. Sea $\left[x_{i}\right]$ el sistema de coordenadas homogéneas inducidas por $\varepsilon$, y para cada $p \in \mathbf{E}-H_{0}$, escribimos: $X_{i}(p)=\frac{x_{j}(p)}{x_{0}(p)} i=1, \ldots, n$. Nótese que $X_{i}(p)$, es la coordenada cartesiana $i$-ésima de $p$, inducida por $\left[x_{i}\right]$ en el espacio afín $\mathbf{E}-H_{0}$.

Para $\alpha>0$, sea $U_{\alpha}(\varepsilon)=\left\{p \in \mathbf{E}-H_{0}:\left|X_{i}(p)\right|<\alpha\right\}$. La familia $\left\{\dot{U_{\alpha}}(\varepsilon): \alpha>0\right\}$, constituye una base de entornos para $e_{0}$, y por ser $D$ abierto, existe $\alpha>0$ con $U=U_{\alpha}(\varepsilon) \subset D$. Reeligiendo si fuera necesario el punto $u$, podemos suponer sin pérdida de generalidad que $\alpha>1$, y por tanto, $u \in U_{\alpha}$.

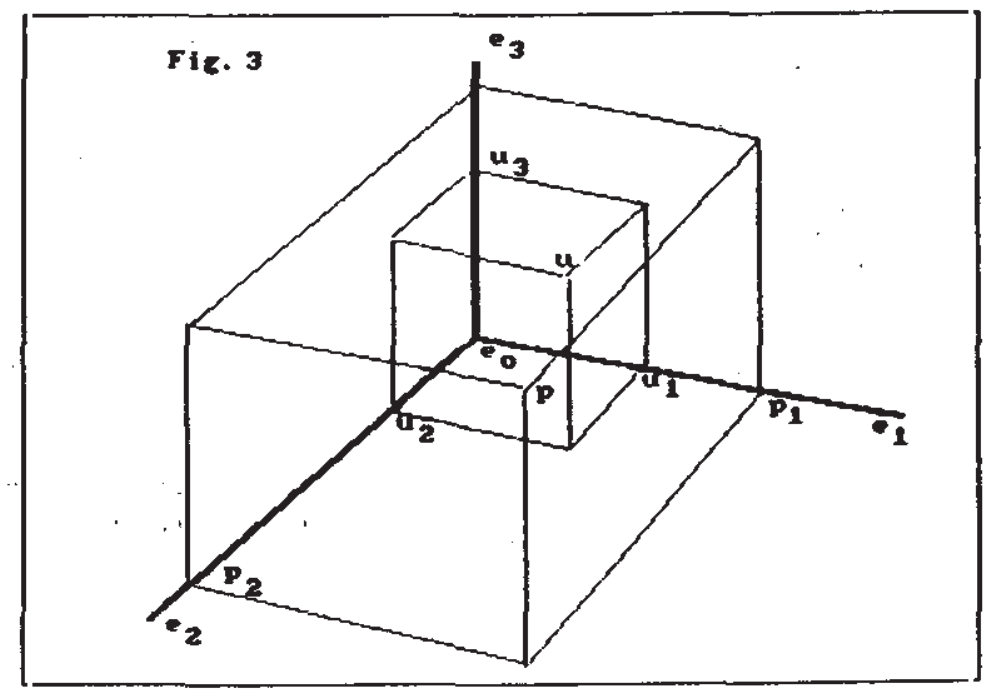

Si $p \in \mathbf{E}-H_{0}$, sea $\left\{\pi_{i}(p)\right\}=<e_{0}, e_{i}>\cap\left(p+H_{i}\right)$ para $i=1, \ldots, n$. Claramente $U$ es invariante por cada $\pi_{i}$, y se verifica la igualdad:

$$
X_{i}(p)=\left[e_{0}, e_{i} ; \pi_{i}(u), \pi_{i}(p)\right]
$$

Por la proposición $2.6, f(\varepsilon)=\left(e_{0}^{\prime}, \ldots, e_{n}^{\prime}, u^{\prime}\right)$ es un sistema de referencia proyectivo contenido en $f(D)$. Como antes, denotamos:

$$
H_{i}^{\prime}=\left\langle e_{0}^{\prime}, \ldots, \hat{e}_{i}^{\prime}, \ldots e_{n}^{\prime}\right\rangle,\left\{\dot{\pi}_{i}^{\prime}\left(p^{\prime}\right)\right\}=<e_{0}^{\prime}, e_{i}^{r}>\cap\left(p^{\prime}+H_{i}^{\prime}\right)
$$


Nuevamente рог 2.6 se deduce que:

$$
f\left(H_{i} \cap U\right)=H_{i}^{\prime} \cap f(U), \text { y } f\left(\left(\mathbf{E}-H_{0}\right) \cap U\right)=\left(\mathbf{E}^{\prime}-H_{0}^{\prime}\right) \cap f(U)
$$

En particular se verifica para todo $p \in U$ :

$$
\begin{aligned}
f\left(\pi_{i}(p)\right)= & f\left(<e_{0}, e_{i}>\cap\left(p+H_{i}\right)\right)=f\left(<\mathrm{e}_{0}, e_{i}>\right) \cap f\left(p+H_{i}\right)= \\
& <e_{0}^{\prime}, e_{i}^{\prime}>\cap\left(f(p)+H_{i}^{\prime}\right)=\pi_{i}^{\prime}(f(p)) \in f(U)
\end{aligned}
$$

Denotando como antes, $\left[x_{i}^{\prime}\right]$ las coordenadas homogéneas respecto a $\varepsilon^{\prime} y$

$$
X_{i}^{\prime}=\left(p^{\prime}\right)=\frac{x_{i}^{\prime}\left(p^{\prime}\right)}{x_{0}^{\prime}\left(p^{\prime}\right)}
$$

se verifica para $p^{\prime}=f(p)$, con $p \in U$;

$$
X_{i}^{\prime}(f(p))=\left[e_{0}^{\prime}, e_{i}^{\prime}, \pi_{i}^{\prime}\left(u^{\prime}\right), \pi_{i}^{\prime}(f(p))\right]=\left[f\left(e_{0}\right), f\left(e_{i}\right) ; f\left(\pi_{i}(u)\right), f\left(\pi_{i}(p)\right)\right]=X_{i}(p) .
$$

Por tanto, $f$ coincide en $U$ con la única homografía $f_{U}$ que transforma $\varepsilon$ en $\varepsilon^{\prime}$. Probemos por último que $f(a)=f_{U}(a)$ para todo $a \in D$. En efecto, si $D_{0}$ denota al interior del conjunto $\left\{a \in D: f(a)=f_{U}(a)\right\}$, se ve que $D_{0}$ es no vacio, pues por ejemplo, $e_{0} \in D_{0}, y$ abierto. Veamos que también es cerrado (relativamente a $D$ ):

Si el punto $a \in D$ pertenece a la adherencia de $D_{0}$, por la misma construcción anterior, podemos suponer que $f$ coincide con cierta homografía $f_{V}$ en cierto entorno $V$ de $a$, y entonces, $f_{U}$ y $f_{V}$ coinciden sobre el abierto no vacío $V \cap D_{0}$. Así, $f_{U}=f_{V}$, y necesariamente $V \subset D_{0}$, y $a \in D_{0}$.

Como $D$ es conexo, $D_{0}=D$, y esto concluye la demostración.

El siguiente resultado, junto con el teorema anterior, constituyen como veremos los puntos clave de la demostración del teorema del cono:

\section{Lema 2.8}

Sea $h: \Delta \rightarrow \Delta$ una involución distinta de la identidad, con puntos fijos $p_{0}$, $p_{\infty}\left(p_{0} \neq p_{\infty}\right)$ de una recta proyectiva real $\Delta$. Supóngase $p_{1} \in \Delta-\left\{p_{0}, p_{\infty}\right\}$, y sea $q_{1} \in \Delta_{1}-\left\{p_{1}\right\}$, siendo $\Delta_{1}$ la componente conexa de $\Delta-\left\{p_{0}, p_{\infty}\right\}$ que contiene $a p_{1}$.

Finalmente sea $S$ un subconjunto de $\Delta_{1}$ verificando las condiciones:

i) $p_{1}, q_{1} \in S$ 
ii) Si $p, q, r$ son tres puntos de $\Delta_{1}$, tales que $[p, h(p) ; q, r]=-1$ y dos de ellos están en $S$, necesariamente está en $S$ el tercero.

Entonces se concluye que $S$ es denso en $\Delta_{1}$.

\section{Demostración: $\quad \therefore$}

El sistema $\left(p_{0}, p_{\infty}, p_{1}\right)$ constituye un sistema de referencia proyectivo en $\Delta$, y proporciona por tanto un sistema de coordenadas homogéneas $\tilde{\mathbf{x}}: \Delta-\tilde{\mathbf{R}}=\mathbf{R} \cup\{\infty\}$, tal que $\tilde{\mathbf{x}}\left(p_{0}\right)=0, \tilde{\mathbf{x}}\left(p_{\infty}\right)=\infty$ y $\tilde{\mathbf{x}}\left(p_{1}\right)=1$.

Por tanto, la restricción $\mathbf{x}$ de $\tilde{\mathbf{x}}$ a la recta afín $\Delta-\left\{p_{\infty}\right\}$ proporciona un isomorfismo afín $\mathbf{x}: \Delta \rightarrow\left\{p_{\infty}\right\} \rightarrow \mathbf{R}$, que define en $\Delta-\left\{p_{\infty}\right\}$ un sistema de coordenadas cartesianas. Denotamos por $\mathbf{R} \ni \lambda \rightarrow p_{\lambda} \in \Delta-\left\{p_{\infty}\right\}$ a la aplicación inversa. Así se tiene la identidad $\mathbf{x}\left(p_{\lambda}\right)=\lambda$, y las ecuaciones de $h$ se escriben: $h\left(p_{\lambda}\right)=p_{-\lambda}$. Además se verifica que $\Delta_{1}=\left\{p_{\lambda}: \lambda>0\right\}$, y como la aplicación x: $\Delta_{1}: p_{\lambda} \rightarrow \lambda \in \mathbf{R}^{+}$es, homeomorfismo, basta demostrar que $S=\left\{\lambda: p_{\lambda} \in S\right\}$ en denso en $\mathbf{R}^{+}$.

Supóngáse $\mathbf{x}(q) \stackrel{+}{*} k$. La propiedad i) se escribe ahora como:

i) $S \subseteq \mathbf{R}^{+}, 1, k \in S$, y $k \neq 1$. Teniendo en cuenta que $\mathrm{x}$ preserva la razón doble se dedúcé a partir de ii), que el conjunto $S$ verifica la propiedad:

ii') Si $x, \lambda, \mu \in \mathbf{R}^{+}, y_{1}[x,-x ; \lambda, \mu]=-1$, entonces si dos elementos de $\{x, \lambda, \mu\}$ están en ' $S$, țambién lo está el tercero.

Como $[x,-x ; \lambda, \mu] \stackrel{=}{=}-1 \Rightarrow x^{2}=\lambda \mu$, las condiciones i') y ii') se traducen a través del logaritmo Neperiano $\log : \mathbf{R}^{+} \rightarrow \mathbf{R}$ sobre el conjunto $\mathbf{T}=\log (S)$ por:

$$
\begin{aligned}
& \text { in }^{\prime \prime} 0, K=\log (k) \leqq \mathbf{T}, 0 \neq K . \\
& \text { ii") Si } a, b, c \in \mathbf{R}, \text { y } a=\frac{b+c}{2} \text { entonces si dos elementos de }\{a, b, c\} \text { están }
\end{aligned}
$$
en $T$, también lo está el tercero.

- El conjunto Tontiene entonces necesariamente a $\left\{\frac{m}{2^{n}} K: m \in \mathbf{Z}, n \in \mathbf{N}\right\}$, por lo' qué es dènso'en $\mathbf{R}$. Así, $S=\exp (T)$ es denso en $\mathbf{R}^{+}, y=\mathbf{x}^{-1}(S)$ es denso en $\Delta_{1}$. 


\section{DEMOSTRACION DEL TEOREMA DEL CONO}

Recordemos previamente su enunciado:

\section{Teorema del cono:}

Si $\mathscr{L}$ es un cono de luz en el espacio proyectivo real $\mathbf{E}$, entonces existe una (única) cuádrica de Lorentz $\mu$ en $\mathbf{E}$ tal que:

$$
\operatorname{im} \mu=\mathscr{C}
$$

\section{Demostración:}

Si $\operatorname{dim} \mathbf{E}=1$, la demostración del teorema del cono es trivial, pues entonces necesariamente $\mathscr{C}$ está formado por dos puntos distintos $a, b \in \mathbf{E}$, que constituyen el conjunto de puntos de una cuádrica de Lorentz con ecuación $-x_{0}^{2}+x_{1}^{2}=0$, siendo $\left[x_{0}, x_{1}\right]$ un sistema de coordenadas homogéneas en el que $a$ tiene por coordenadas $[1,1]$ y $b[1,-1]$. Nótese que en este caso el conjunto $\mathscr{D}$ de sus centros, coincide con $\mathbf{E}-\{a, b\}$.

Se supondrá de ahora en adelante que dim $\mathbf{E}$ es mayor o igual a dos, y para cada $p \in \mathscr{Q}(\mathscr{D}$ es el conjunto de centros) se denotará por $\mathbf{H}(p)$ al hiperplano central de $p$, y por $\varphi_{p}: \mathbf{E} \rightarrow \mathbf{E}$ la homologia involutiva de centro $p$ e hiperplano central $\mathbf{H}(p)$. Recuérdese que tal homologia viene definida por la condición:

$$
\left[p,\left\langle p, x>\cap \mathbf{H}(p) ; \varphi_{p}(x), x\right]=-1\right.
$$

para todo $x \in \mathbf{E}-(H(p) \cup\{p\})$, dejando fijos los puntos de $H(p) \cup\{p\}$.

La restricción de $\varphi_{p}$ al espacio afín $\mathbf{E}-H(p)$ es la simetría afín de centro $p$, que por hipótesis deja invariante el cono de luz $\mathscr{C}$.

Por hipótesis $\mathscr{D}$ contiene a cierto conjunto $D$ abierto que podemos suponer conexo:

Fijemos $p_{1} \in D$ y $\Delta$ una recta proyectiva que pasa por $p_{!}$. La recta $\Delta$ corta a $\mathscr{C}$ en exactamente dos puntos distintos $p_{0}, p_{\infty}$. Denotemos por $\Delta_{1}$ a la componente conexa de $\Delta-\left\{p_{0}, p_{\infty}\right\}$ que contiene al punto $p_{1}$, y sea $q_{1} \in\left(\Delta_{1}-\left(p_{1}\right\}\right) \cap D$.

Tomemos $L=H\left(p_{1}\right) \cap H\left(q_{1}\right)$, sea $S=\left\{s \in \Delta_{1} \cap \mathscr{Z}: L \subset H(s)\right\}$, y sea $L^{\omega}=\left\{H \in \mathrm{E}^{*}: L \subset H\right\}$, que es recta proyectiva de $\mathrm{E}^{*}$, y finalmente sea $h: \Delta \rightarrow \Delta$ la involución hiperbólica con puntos fijos $\left\{p_{0}, p_{\infty}\right\}$. 
En estas condiciones probaremos el siguiente resultado:

\section{Lema}

El conjunto $S$ coincide con $\Delta_{1}$, y el siguiente diagrama es conmutativo:

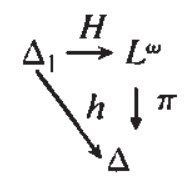

Siendo $\pi$ la homografia $\pi: L^{\omega} \ni H \rightarrow \Delta \cap H \in \Delta$. En particular, la aplicación:

$$
\Delta_{1} \ni p \rightarrow \dot{H}(p) \in L^{\omega}
$$

preserva la razón doble:

\section{Demostración:}

a) Probaremos, que el conjunto $S$ es denso en $\Delta_{1}$.

Verificaremos la hipótesis ii) del lema 2.7 para la involución hiperbólica $h$ y el subconjunto $S$. Esto es suficiente para probar la afirmación a):

Si $p, q, r$ son tres puntos de $\Delta_{1}$ tales que $[p, h(p) ; q, r]=-1$, y supuesto por ejemplo que $p, q \in S$, como $H(p) \cap \Delta=h(p)$, se concluye que $\varphi_{p}(q)=r, \mathrm{y}$ $\varphi_{p}(r)=\varphi_{p}^{2}(q)=q$. Por otra parte, $r \in \mathscr{D}$. En efecto:

Si $R$ es una recta proyectiva que pasa por $r$, entonces $\varphi_{p}(R)$ es una recta proyectiva que pasa por $q$, y como $q \in \mathscr{P}$, corta a $\mathscr{C}$ en $q_{0}$ y $q_{\text {oo }}$ con $q_{0} \neq q_{\infty}$. Así $R \cap \mathscr{C}=\varphi_{p} \varphi_{p}(R) \cap \mathscr{C}=\left\{\varphi_{p}\left(q_{0}\right), \varphi_{p}\left(q_{\infty}\right)\right\}$.

Finalmente, si $\varphi$ es la homologia involutiva de centro $r$ e hiperplano central $\varphi_{p}(H(q))$, se ve que $\varphi=\varphi_{p} \cdot \varphi_{q} \cdot \varphi_{p}$, y $\varphi$ es composición de aplicaciones que dejan invariante a $\mathscr{C}$. Así, $\varphi$ deja invariante $\mathscr{C}, r \in \mathscr{Q}$, y $H(r)=\varphi_{p}(H(q))$.

Como $L=H(p) \cap H(q), \varphi_{p}$ deja fijos los puntos de $L$, y $L=\varphi_{p}(H(q))=$ $H(r)$. Esto prueba que $r \in S_{i}$.

b) Probaremos ahora que $S$ es exactamente igual a $\Delta_{1}$ : (Véase figura 4).

Fijado un punto $q \in \Delta_{1}$ tomemos una sucesión $\left(q_{i}\right) \subset S$ con $\lim _{i \rightarrow \infty} q_{i}=q$. Los hiperplanos $H\left(q_{i}\right)$ contienen a $L$ y al punto $h\left(q_{i}\right)$. Sea $H_{a}$ el hiperplano que 
contiene a $L$ y a $h(q)$. Demostraremos que $q \in \mathscr{Z}$ y $H(q)=H_{q}$. En efecto, si $Q$ es una recta proyectiva que pasa por $q, y\{x\}=Q \cap H_{q}$, se puede tomar una sucesión $\left(x_{i}\right)$ con $x_{i} \in H\left(q_{i}\right)$ y $\lim _{i=\infty} x_{i}=x$. Las rectas $<q_{i}, x_{i}>$ cortan a $\mathscr{C}$ en dos puntos distintos $q_{i}^{+}, q_{i}^{-}$de forma que $\varphi_{q_{i}}\left(q_{i}^{+}\right)=q_{i}^{-}$, es decir:

$$
\left[q_{i}, x_{i}, q_{i}^{+}, q_{i}^{-}\right]=-1
$$

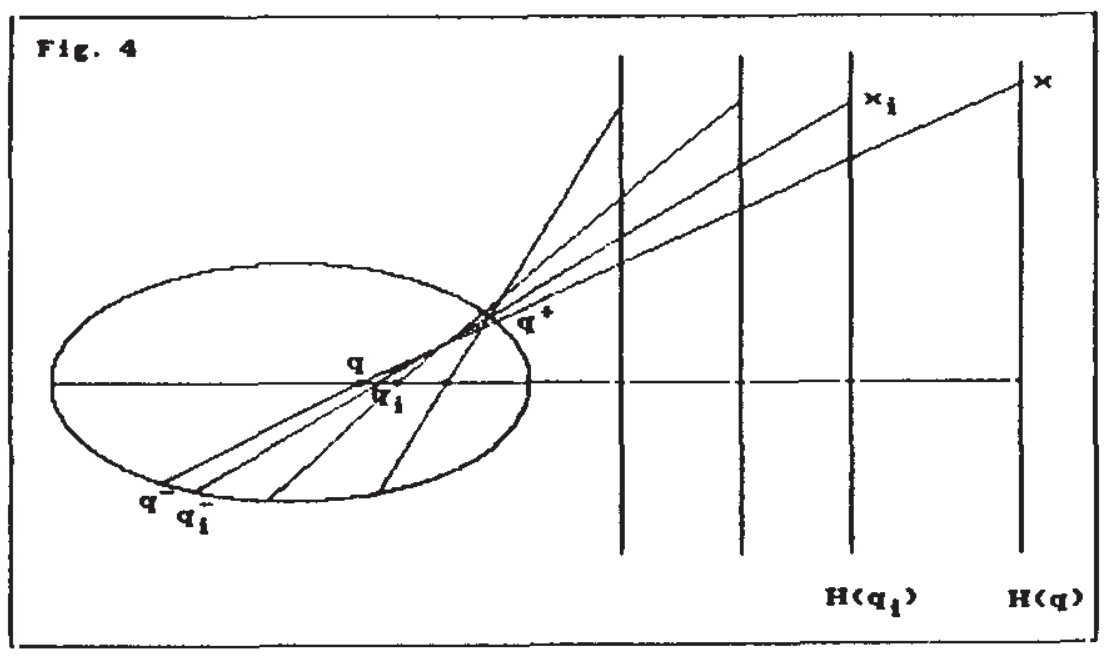

Como $\mathscr{C}$ es compacto, podemos suponer (quizás tomando subsucesiones) que $\left(q_{i}^{+}\right)$converge a cierto punto $q^{+} \in \mathscr{C}$, y $\left(q_{i}\right)$ a $q-\in \mathscr{C}$. Los puntos $q^{+}$y $q^{-}$ están también en la recta $\langle x, q\rangle=Q$, por lo que $\left\{q^{+}, q^{-}\right\} \subseteq Q \cap \mathscr{C}$.

Por otra parte, tomando límites queda:

$$
\left[q, x ; q^{+}, q^{-}\right]=-1
$$

esto prueba en particular que $q^{+} \neq q^{-}$, que $Q \cap \mathscr{C}=\left\{q^{+}, q^{-}\right\}, q \in \mathscr{D}$ y $H_{q}=H(q)$

c) Para probar la conmutatividad del diagrama, es suficiente observar que para cada $q \in \Delta_{1}$ se verifica que $H(q) \cap \Delta=h(q)$, ya que $\left[q, h(q) ; p_{0}\right.$, $\left.p_{\infty}\right]=-1$.

La demostración del teorema del cono, se concluye de la siguiente forma:

Sea $p_{1}$ un punto interior a $\mathscr{D}$. Para cada recta $\Delta$ que pasa por $p_{1}$ denotamos por $\Delta_{1}$ a la componente conexa de $\Delta-(\Delta \cap \mathscr{C})$ que contiene a $p_{1}$. Sea 
$\mathscr{D}\left(p_{1}\right)$ el conjunto formado por la unión de todos los segmentos $\Delta_{1}$ cuando $\Delta$ recorre todas las rectas proyectivas que pasan por $p_{1}$. Entonces $\mathscr{B}\left(p_{1}\right)$ es un abierto conexo, y por el lema anterior, $\mathscr{Q}\left(p_{1}\right) \subseteq \mathscr{Q}$ y la aplicación:

$$
H: \mathscr{D}\left(p_{1}\right) \ni p \rightarrow H(p) \in \mathbf{E}^{*}
$$

preserva la razón doble.

Por el Teorema 2.7, existe una homografía única $\bar{H}: \mathbf{E} \rightarrow \mathbf{E}^{*}$ con $\bar{H} \mid \mathscr{D}\left(p_{1}\right)=H$.

Para ver que $\bar{H}$ es una polaridad, es suficiente comprobar que para todo punto $p \in \mathscr{D}\left(p_{1}\right)$ se verifica la equivalencia:

$$
q \in H(p) \Longleftrightarrow p \in H(q)
$$

Si $p \in \mathscr{O}\left(p_{1}\right)$, entonces $p \in \mathscr{\mathscr { D }}$, y por. tanto $p \in \mathbf{E}-H(p)$, Supóngase $q \in H(p)$, y sea $\Delta$ la recta que une $p$ y $q . \bar{H}(\Delta)$ 'es una recta proyectiva de $\mathbf{E}^{*}$, y puede' describirse en la forma $H(\Delta)=L^{\omega}$ para cierto subespacio $L$ de codimensión 2 en $\mathbf{E}$. Denotando por $h: \Delta \rightarrow \Delta$ la involución hiperbólica con puntos fijos $\Delta \cap \mathscr{C}=\left\{p_{0}, p_{\infty}\right\}$, y por $\Delta_{1}$ la componente conexa de $\Delta-\left\{p_{0}, \dot{p}_{\infty}\right\}$ que contiene a $p$, por el Lema, se verifica la conmutatividad del diagrama:

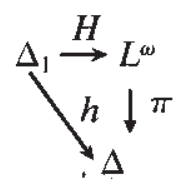

Por ser $\bar{H}$ homografía extensión de $\mathbf{H}$, se concluye que también conmuta el diagrama:

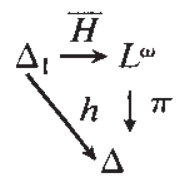

así, como $q \in \bar{H}(p) \cap \Delta$ es $q=h(p)$, y al ser $h$ involutiva se tiene $h(q)=p$, es decir, $p \in H(q) \cap \Delta \subset H(q)$. Recíprocamente, si $p \in \widetilde{H}(q)$ entonces $q \in \bar{H}(p)$.

La polaridad $\frac{1}{H}$ viene inducida entonces por una única cuádrica $\mu$ con

$$
\operatorname{im} \mu=\{p \in \mathbf{E}: p \in \bar{H}(p)\}
$$

Claramente $\mathscr{C}$ está contenidọ en im $\mu$ y los puntos de $\mathscr{D}$ son interiores a $\mu$. Así, $\mu$ es una cuádrica de Lorentz. Por otra parte, im $\mu \subseteq \mathscr{C}$, ya que si 
existe $p \in \operatorname{im} \mu-\mathscr{C}$, tomando $p_{1} \in \mathscr{P}$ la recta $\Delta=\left\langle p, p_{1}\right\rangle$ cortaría a im $\mu$ en tres puntos distintos $p_{0}, p_{\infty}$, y $p$, siendo $\Delta \cap \mathscr{C}=\left\{p_{0}, p_{\infty}\right\}$.

\section{APENDICE: SOBRE LOS FUNDAMENTOS GEOMETRICOS DE LA RELATIVIDAD ESPECIAL}

\subsection{La cỉnemática Galileana}

La cinemática clásica de partículas está fundamentada en la existencia de un espacio (euclideo) y un tiempo absolutos, junto con el principio de relatividad de Galileo (invariancia de las leyes físicas frente al movimiento inercial).

El modelo matemático adecuado para su estudio lo denominamos espacio de Galileo, y viene dado por un espacio afín real $\mathbf{M}$ de dimensión 4 , que representa el espacio de sucesos, y un hiperplano vectorial $S$ del espacio vectorial $\overrightarrow{\mathbf{M}}$ (dirección de $\mathbf{M}$ ), que se denomina subespacio de simultaneidad. Si $S$ está dotado de una métrica euclidea $g$, se denomina a $\mathbf{M}=(\mathbf{M}, S, g)$ espacio euclideo de Galileo.

En un espacio de Galileo, dos sucesos $a, b \in \mathbf{M}$ se consideran simultáneos si $\overrightarrow{a b} \in S$. El espacio de sucesos $\mathbf{M}$ queda foliado asi por hiperplanos afines de simultaneidad de la forma $a+S$ con $a \in \mathbf{M}$. Cada uno de estos hiperplanos representa nuestro espacio ordinario considerado en un cierto instante de tiempo.

Desde el punto de vista físico $S$ representa el concepto de tiempo universal de la cinemática clásica. Una medida de este tiempo es desde el punto de vista geométrico una aplicación afín $\mathbf{t}: \mathbf{M} \rightarrow \mathbf{R}$ tal que $\mathbf{t}^{-1}(\lambda)$ es un hiperplano afín de simultaneidad para todo $\lambda \in \mathbf{R}$.

La aplicación lineal asociada a $\mathbf{t}, \overrightarrow{\mathbf{t}}: \overrightarrow{\mathbf{M}} \rightarrow \mathbf{R}$ verifica ker $\overrightarrow{\mathbf{t}}=S$, y puede entenderse como un isomorfismo lineal $\overrightarrow{\mathrm{t}}: \overrightarrow{\mathbf{M}} / S \rightarrow \mathbf{R}$. A un isomorfismo lineal $\mathbf{u}: \overrightarrow{\mathbf{M}} / S \rightarrow \mathbf{R}$ se le denomina unidad de tiempo. $\mathbf{u}^{-1}\left(\mathbf{R}^{+}\right)$es una de las dos componentes conexas de $(\overrightarrow{\mathbf{M}} / S)-\{0\}$. Tal elección se corresponde con la idea física de sentido del tiempo, y se denomina orientación temporal inducida por la unidad de tiempo u.

Nótese que una unidad de tiempo u viene univocamente determinada, por la elección de la hoja $\mathbf{u}^{-1}(1) \in(\overrightarrow{\mathbf{M}} / S)-\{0\}$ o bien por la elección de un vector $u_{1} \in \mathbf{u}^{-1}(1)$. Por otra parte, una media del tiempo $t$ queda determinada cuando se conoce un suceso inicial $a_{0} \in \mathbf{M}\left(\operatorname{con} \mathbf{t}\left(a_{0}\right)=0\right)$ y la unidad de tiempo $\overrightarrow{\mathbf{t}}$. 
Una partícula material, describe en $\mathbf{M}$ una trayectoria $P$ (no parametrizada a priori) que determina su historia, y se denomina línea del universo de la partícula. $P$ es por definición una subvariedad unidimensional transversal a los hiperplanos de simultaneidad. En particular, las líneas del universo que son rectas, se denominan (observadores o) particulas inerciales.

Los observadores inerciales, y el tiempo universal $S$ determinan completamente la estructura afín, y, por tanto, el espacio $\mathbf{M}$ de Galileo.

Sea $A$ un observador inercial, es decir, $A$ es una recta afín cuya dirección $\vec{A}$ es transversal a $S$. El espacio vectorial $\overrightarrow{\mathbf{M}}$ se descompone entonces en la forma: $\overrightarrow{\mathbf{M}}=\vec{A} \oplus S$.

Sea $\pi_{A}: \mathbf{M} \rightarrow A$ la proyección paralela con base $A$ y dirección $S$, es decir: $\pi_{A}(p)=(p+S) \cap A$ para cada $p \in \mathbf{M}$. Se denomina posición del suceso $p \in \mathbf{M}$ respecto a $A$, al vector $\Pi_{A}(p)=\overline{\pi_{A}(p) p}$. Nótese que la aplicación $\Pi_{A}: \mathbf{M} \rightarrow S$ es afín, y su lineal asociada $\vec{\Pi}_{A}: \overrightarrow{\mathbf{M}} \rightarrow S$ coincide con la proyección vectorial sobre $S$ con dirección $\vec{A}$. Si $P$ es una partícula inercial, al conjunto $\Pi_{A}(P)$ se le denomina trayectoria espacial de $P$ respecto a $A$.

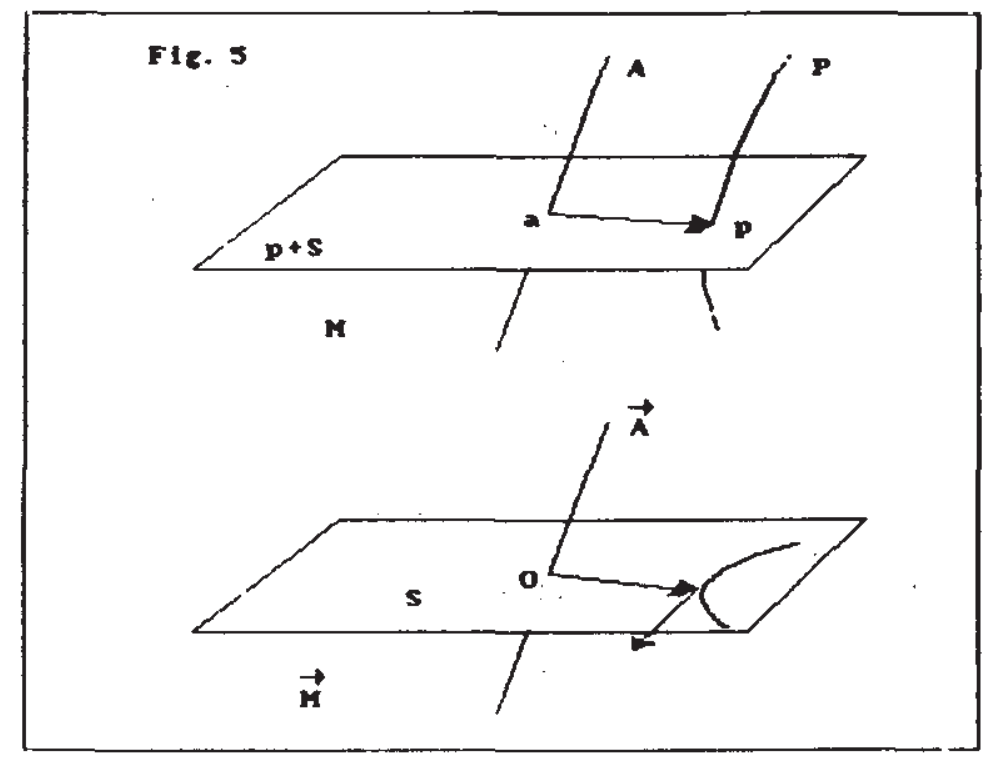

Una medida del tiempo $t: \mathbf{M} \rightarrow \mathbf{R}$, induce sobre la partícula material $P$ un difeomorfismo $t_{P}: P \rightarrow \mathbf{R}$, cuya inversa $t_{P}^{-1}=\gamma_{P}: \mathbf{R} \ni \tau \rightarrow \gamma_{P}(\tau) \in P$ es una carta global de $P$, que se denomina parametrización temporal de $P$. 
Las aplicaciones $\Pi_{A} \cdot \gamma_{P}: \mathbf{R} \rightarrow S$ y su derivada $\left(\Pi_{A} \cdot \gamma_{P}\right)^{\prime}: \mathbf{R}-S$ establecen respectivamente la posición y la velocidad respecto a $A$ de la partícula $P$ en función del tiempo $t$. Denotamos $A P(\tau)=\left(\Pi_{A} \cdot \gamma_{P}\right)(\tau)$ y $A \vec{P}(\tau)=\left(\Pi_{A} \cdot \gamma_{P}\right)^{\prime}(\tau)$ para $\tau \in \mathbf{R}$. Si nuestro espacio de Galileo es euclídeo, al número $\|\overrightarrow{A P}(\tau)\|$ se le denomina velocidad escalar de $P$ respecto a $A$ en el instante $\tau$.

Se tiene entonces el siguiente resultado, que es independiente de la medida del tiempo $t$ elegida, y tiene demostración elemental:

\section{Teorema}

La particula $P$ es inercial, si y sólo si la velocidad $\overrightarrow{A P}: \mathbf{R} \rightarrow S$ es constante. (Se denota por $\overrightarrow{A P}=\overrightarrow{A P}(\tau)$ a dicho vector constante). En este caso, la aplicación $A P: \mathbf{R} \rightarrow \Pi_{A}(P) \subset S$ define una parametrización afin de la trayectoria espacial $\Pi_{A}(P)$.

En particular, las particulas inerciales $B$ tales que $\overrightarrow{A B}=0$, se dicen en reposo respecto a $A$ (su trayectoria espacial $\Pi_{A}(B)$ se reduce a un punto), $y$ vienen caracterizadas por ser rectas afines paralelas a $A$, es decir, $\vec{A}=\vec{B}$.

Si $A, B$, y $C$ son partículas inerciales se verifica: $\overrightarrow{A B}+\overrightarrow{B C}=\overrightarrow{A C}$, y esto expresa la regla de adición Galineana de velocidades.

\subsection{Espacio de velocidades}

Las direcciones de las partículas inerciales se identifican con puntos del espacio $\mathbf{V}=\mathbf{P}(\overrightarrow{\mathbf{M}})-\mathbf{P}(S)$ que es un espacio afín, por ser complementario del hiperplano $\mathbf{P}(S)$ en el espacio proyectivo $\mathbf{P}(\overrightarrow{\mathbf{M}})$. Se denomina a $\mathbf{V}$ espacio de velocidades. Si $v, w \in \mathbf{V}$, el vector alibre" $\overrightarrow{v w}$ definido por ellos, representa la velocidad relativa de $B$ respecto a $A$, cuando $A$ y $B$ son dos partículas inerciales que definen las direcciones $v, \mathrm{y} w$ respectivamente. Este vector, no depende en principio de la medida del tiempo $t$, pero una vez elegida, se identifica con el vector $\overrightarrow{A B}$, a través del isomorfismo afín $V \ni \nu \rightarrow \nu \cap S_{1} \in S_{1}$, donde $S_{1}=\bar{t}^{-1}$ (1) tiene estructura afín canónica sobre $S=\operatorname{ker}(t)$. Si nuestro espacio de Galileo es euclídeo, entonces $S_{1}, \mathrm{y}$, por tanto, $\mathrm{V}$, se transforma en un espacio afín euclídeo, y $\|\overrightarrow{A B}\|$ es la velocidad escalar constante de $B$ respecto a $A$.

Nótese que la estructura afín euclídea del espacio de velocidades, depende de la unidad de tiempo $\bar{t}$, y no de la medida del tiempo $t$ asociada. 


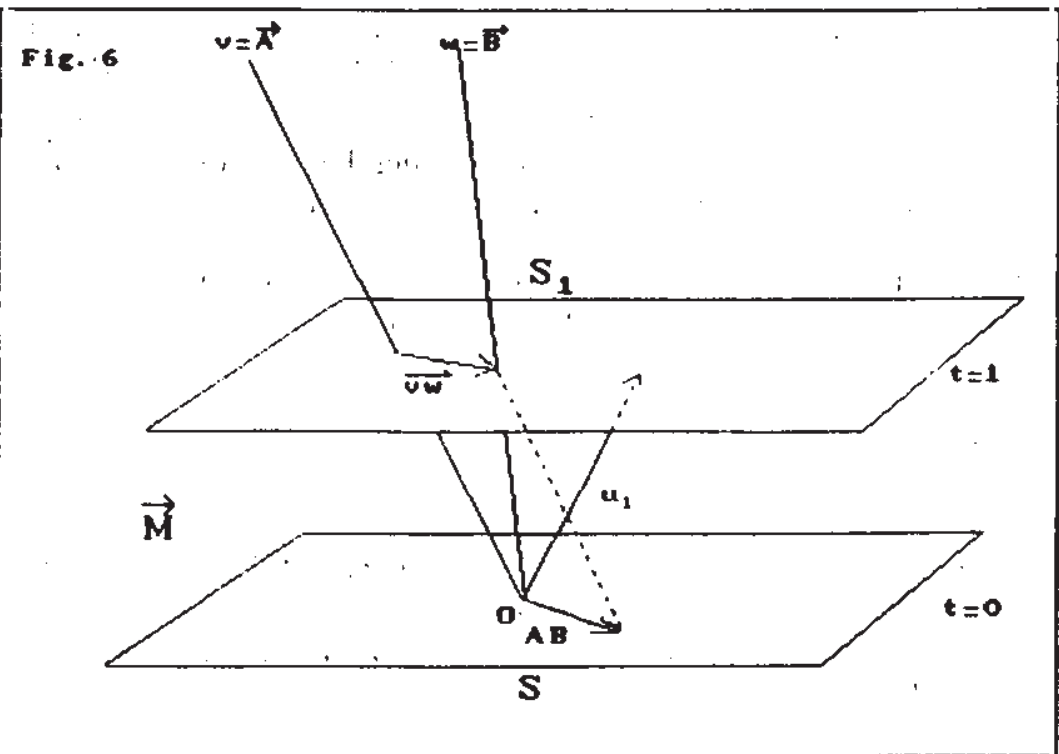

\subsection{EI Modelo Aristoté̉ico}

Un espacio Aristotélico es una terna $\mathscr{A}=(\mathbf{M}, S, \omega)$, donde $(\mathbf{M}, S)$ es un espacio Galileo, y $\omega$ es un punto del espacio afín de velocidades $\mathbf{V}=\mathbf{P}(\overrightarrow{\mathbf{M}})-\mathbf{P}(S)$.

Denominamos a $\omega$ referencia de reposo. Las partículas inerciales $A$, con dirección $\omega$, se llaman observadores (en reposo) de $\mathscr{L}$.

El espacio Aristotélico es, pues, un espacio de Galileo "personalizado". Aquí, el concepto de ureposo absoluto» entra a formar parte de la estructura, rompiendo asi el principio de relatividad de Galileo.

Un subconjunto $\mathscr{C}$ de $\mathbf{P}(\overrightarrow{\mathbf{M}})$ se llama radiación en $\mathscr{A}$ si $\omega$ es punto central de $\mathscr{C}$ con hiperplano central $S$ (ver definición 1.3 a). En particular, $\mathscr{C} \subset \mathrm{V}$.

Por ejemplo, el conjunto de rectas vectoriales (puntos de $\mathbf{P}(\overrightarrow{\mathbf{M}})$ ) que contornean el paralelogramo dibujado en el plano $S_{1}$ de la figura 7 , determinan una radiación en $(\mathbf{M}, S, \omega)$, ya que cada recta afín de $S_{\mathrm{I}}$ que pasa por $\omega_{1}$ (intersección de $\omega$ con $S_{1}$ ) corta en dos puntos al paralelogramo, que son simétricos respecto a $\omega_{I}$. 


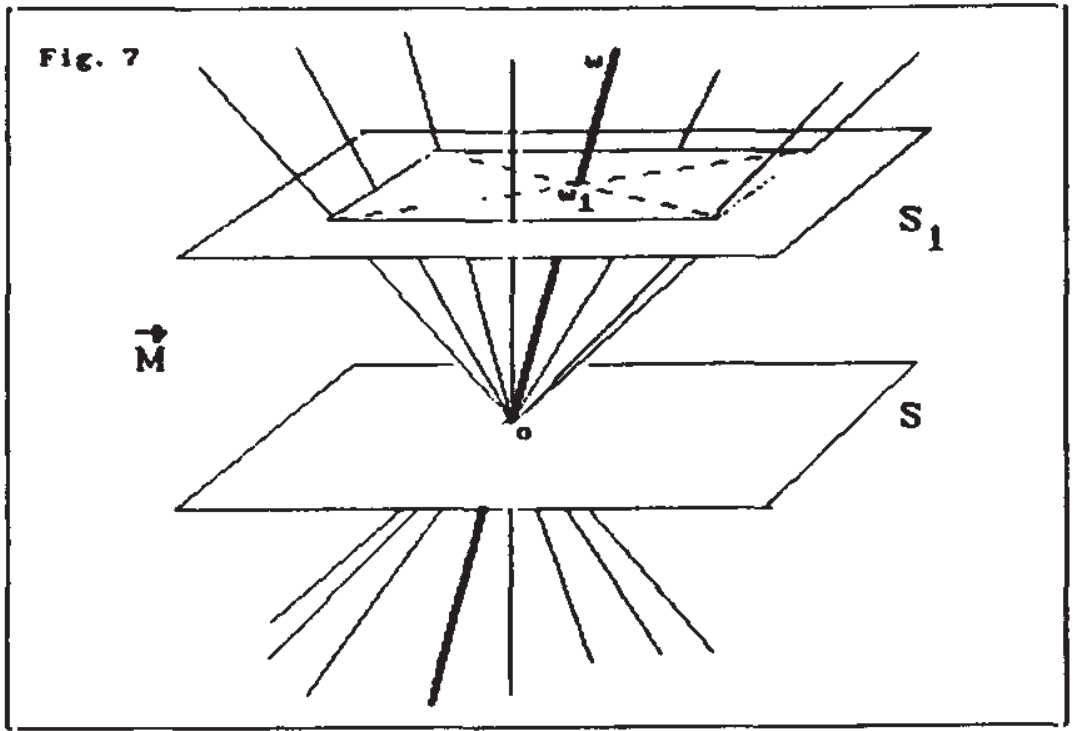

Un rayo (de $\mathscr{C}$ ), es una partícula inercial $R$, cuya dirección $v=\vec{R}$ pertenece a $\mathscr{C}$. Respecto a un observador en reposo $A$ de $\mathscr{A}$ la velocidad de $R$ viene medida por el vector $\overrightarrow{\omega v}$. Como $\omega$ es punto central de $\mathscr{L}$, las partículas inerciales $R^{\prime}$ con dirección $v^{\prime}$ tal que $-\overrightarrow{\omega v}=\overrightarrow{\omega v}$ ' (es decir, con velocidad (opuesta») también son rayos de $\mathscr{C}$.

Una radiación en $\mathscr{A}$ se puede caracterizar por la siguiente propiedad:

Dado un suceso $a \in \mathbf{M}$, un observador en reposo $A$ con $a \in A, y$ una recta vectorial $U$ de $S$, existen exactamente dos rayos de $\mathscr{C}, R$ y $R$ ' que pasan por el suceso a, con direcciones $\vec{R}=v, y \vec{R}^{\prime}=v^{\prime}$ tales que: $\Pi_{A}(R)=\Pi_{A}\left(R^{\prime}\right)=U, y$ $\overrightarrow{\omega v}=-\overrightarrow{\omega v}$.

En estas condiciones, el observador $A$, se ve en cada instante, como punto medio del segmento que une los rayos $R$ y $R$ '. De forma más precisa:

Para cada $p \in A, p$ es el punto medio del segmento (en el espacio de simultaneidad $p+S)$ definido por $(p+S) \cap R$ y $(p+S) \cap R^{\prime}$. (Véase figura 8.)

Nótese por otra parte, que el rayo $R^{\prime}$ es el transformado de $R$ por la simetría afín con base $a+S$ y dirección la referencia de reposo $\omega$.

Si se interpretan los rayos de $\mathscr{C}$ como señales fácilmente reproducibles en cualquier momento y lugar, la condición anterior implica que dichas señales pueden ser usadas por el observatorio aristotélico $\mathscr{A}$ para "reconstruìr" su espacio de simultaneidad $S$. En efecto: 


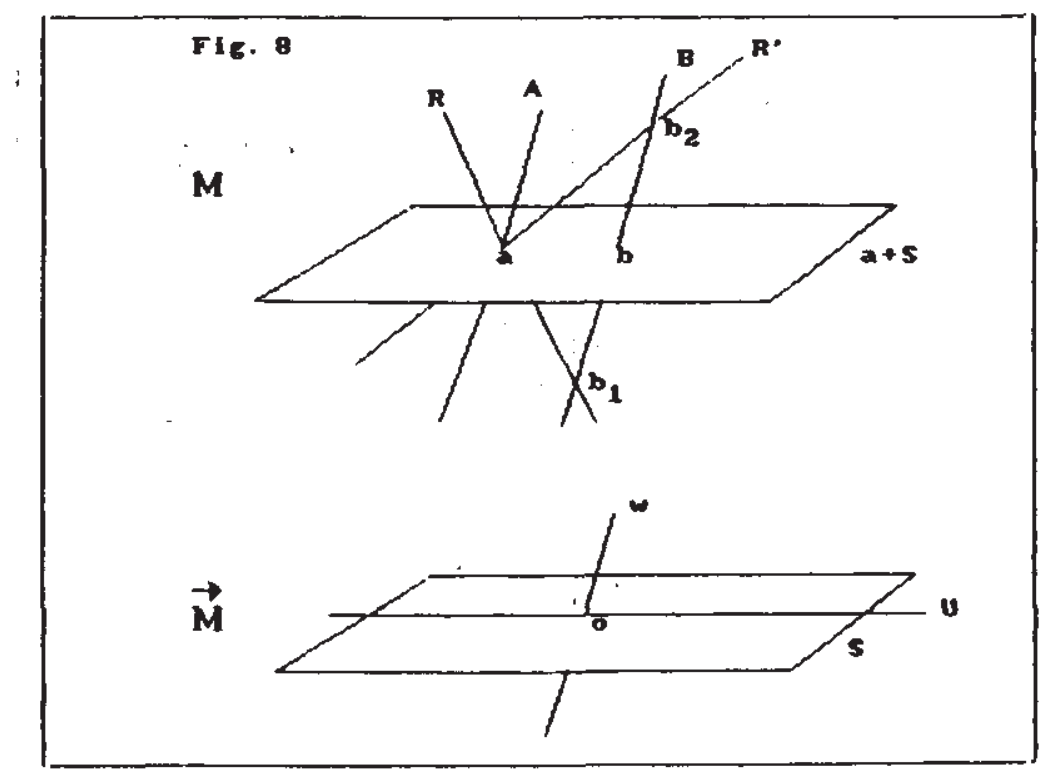

El rayo $R$ de la figura 7 representa una señal enviada por el observador en reposo $B$ en el momento $b_{1} \in B$, que es reflejada por el observador en reposo $A$, en el momento $a \in A$ siguiendo $R$ ', y recibida de nuevo pór $B$ en el suceso $b_{2} \in B$.

El observador $B$, interpretará que el suceso $a \in A$ es simultáneo con el suceso $b=(a+S) \cap B$, pues es el punto medio del segmento $b_{1}$ y $b_{2}$.

Nótese que el concepto de radiación aqui establecido, es estrictamente afin, y no requiere el uso de estruciura euclídea sobre el espacio de simultaneidad.

\subsection{Sobre el Principio Fundamental de la Relatividad Especial}

El Principio Fundamental de la Relatividad Especial establece que la velocidad (iescalar!) $c$ de la luz es la misma independientemente del observatorio inercial desde el cual sea medida.

Esta afirmación presupone implícitamente la existencia de una estructura euclídea sobre el espacio $\mathbf{V}$ de las velocidades correspondiente a cada posible perspectiva Aristotélica $\mathscr{A}=(\mathrm{M}, S, \omega)$.

Una reformulación de este principio, que no exige estructura euclídea previa, y que como veremos conduce a la misma teoría, podría inicialmente enunciarse asi: 
La luz determina una radiación, independientemente del laboratorio inercial desde el cual sea observada.

De hecho, sería suficiente exigir que esta propiedad fuera satisfecha por un "entorno abierto» de observadores inerciales. De forma más precisa:

Existe una radiación $\mathscr{C}$ (la definida por los rayos de luz) respecto a cierto observatorio Aristotélico $\mathscr{Z}=(\mathbf{M}, S, \omega)$, verificando la siguiente propiedad:

Hay un entorno $U$ de $\omega$ en $\mathbf{P}(\overrightarrow{\mathbf{M}})$, tal que para cada $\omega^{\prime} \in U$, existe $S^{\prime \prime}$ hiperplano de $\overrightarrow{\mathbf{M}}$, de forma que en el espacio Aristotélico $\mathscr{A}^{\prime \prime}=\left(\mathbf{M}, S^{\prime}, \omega^{\prime}\right), \mathscr{C}$ sigue siendo radiación. 1.3 .

Se concluye asi que $\mathscr{C}$ debe ser un cono de luz en $\mathbf{P}(\overrightarrow{\mathbf{M}})$ según la definición

El teorema del Cono probado en $\$ 1$ demuestra que en estas condiciones existe una única cuádrica de Lorentz en $\mathbf{P}(\overrightarrow{\mathbf{M}})$ de forma que $\mathscr{L}=$ im $\mu$.

En particular, para todo $\omega \in$ int $\mu$, si $\omega^{\perp}=\mathbf{P}(S)$, entonces en el espacio Aristotélico $\mathscr{R}=(\mathbf{M}, \omega, S), \mathscr{C}$ es una radiación.

Llegamos así al siguiente:

\subsection{Modelo de Minkowski para la relatividad especial}

El modelo de Minkowski consta de un espacio afin real de dimensión cuatro $\mathbf{M}$ (espacio de sucesos), dotado de una forma cuadrática, que define una cuádrica de Lorentz $\mu$ en el infinito $\mathbf{P}(\overrightarrow{\mathbf{M}})$.

En este modelo, un observador inercial viene definido por una recta afin $A$ de $\mathbf{M}$, cuya dirección $\omega=\vec{A}$ pertenece al interior de la cuádrica int $\mu$. Al observador $A$ se le asocia entonces el espacio Aristotélico $\mathscr{A}_{\omega}=(\mathbf{M}, \omega, S)$, siendo $\mathbf{P}\left(S_{\omega}\right)$ el hiperplano de $\mathbf{P}(\overrightarrow{\mathbf{M}})$ conjugado de $\omega$ respecto $\mu$. Se entiende que todos los observadores con dirección $\omega$, realizan sus observaciones cinemáticas usando el espacio $\mathscr{Q}_{\omega}$, según se analizó en $4.1,4.2$ y 4.3 . En particular, una medida del tiempo $t: \mathbf{M} \rightarrow \mathbf{R}$ en $\mathscr{L}_{\omega}$ se denomina tiempo propio de $\omega$, y una unidad de tiempo propio, u: $\overrightarrow{\mathbf{M}} \rightarrow \mathbf{R}$ viene univocamente determinada por un vector $\omega_{1} \in \overrightarrow{\mathbf{M}}$, tal que $\left[\omega_{1}\right]=\omega, \mathrm{y} \mathbf{u}\left(\omega_{1}\right)=1$.

La cuádrica $\mu$ restringida a $\mathbf{P}\left(S_{\omega}\right)$ es una cuádrica euclídea, que determina en $S_{\omega}$ un producto escalar euclídeo $g$, salvo constantes multiplicativas positivas no nulas. Fijar $g$ significa fijar la unidad de longitud. 


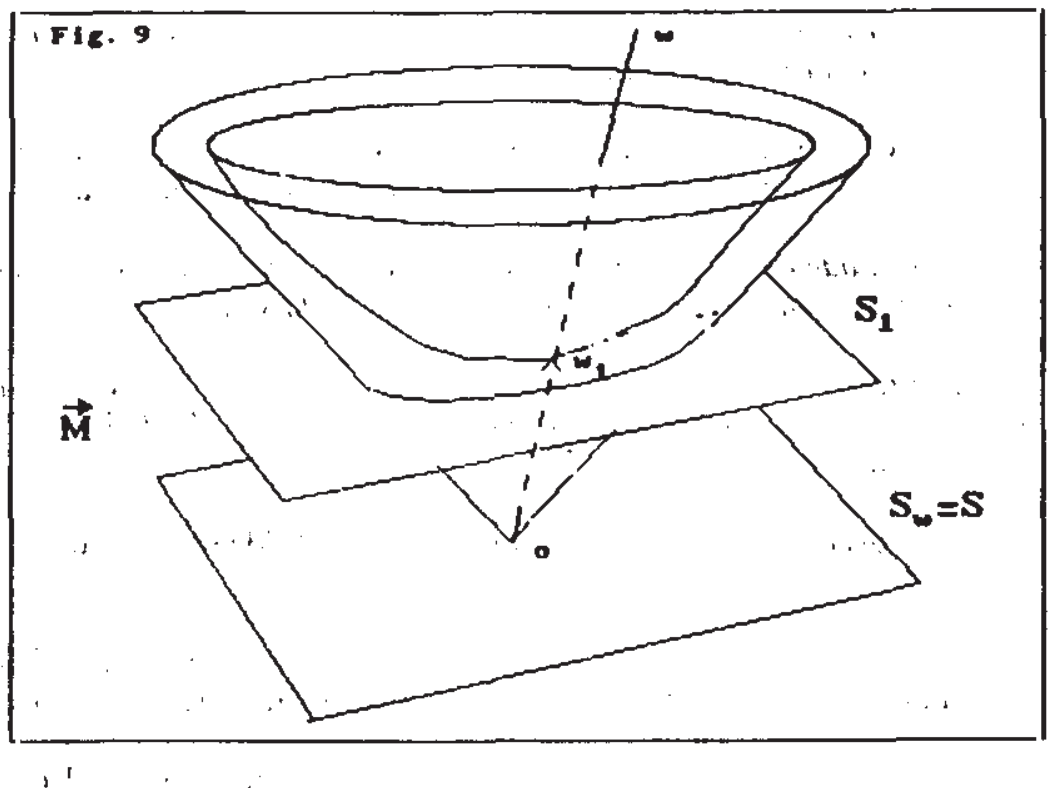

El conjunto $\mathscr{C}=\mathrm{im} \mu$ es la radiación luminosa, y es observada como tal radiación (según definición de 4.3 ) desde cada perspectiva Aristotélica $\_\mathscr{Z}_{\omega}$.

Los rayos de la radiación se denominan ahora rayos de luz, y vienen definidos por rectas afines de $\mathbf{M}$, cuya dirección pertenece a $\mathscr{C}$.

La unidad de tiempo propio $\mathbf{u}, \dot{y}$ la unidad de longitud $g$, permite definir una estructura euclídea en el espacio afín de velocidades $\mathbf{V}_{w}=\mathbf{P}(\overline{\mathbf{M}})-\mathbf{P}\left(S_{w}\right)$ (ver 4.2 ), y el conjunto $\mathscr{C}$, puede escribirse entonces de la forma: $\mathscr{C}=\left\{v \in \mathbf{V}_{\omega}\right.$ : $\|\vec{\omega}\|=c\}$, siendo $c$ una constante positiva, que representa la velocidad escalar constante de los rayos de luz, medidos desde la perspectiva de $\mathcal{Q}_{\omega^{*}}$.

Si $\omega_{1} \in \overrightarrow{\mathbf{M}}$, es tal que $\left[\dot{\omega_{i}}\right]=\dot{\omega}$, y $\mathbf{u}\left(\omega_{1}\right)=1$, la forma cuadrática $q$ en $\overrightarrow{\mathbf{M}}$ que induce sobre $S_{\omega}$ la unidad de longitud $g$, hace a $\omega$ ortogonal a $S_{\omega}$, y toma valor $q\left(\omega_{\mathrm{I}}\right)=-c^{2}$, es una forma cuadrática del Lorentz que induce en $\mathbf{P}(\overrightarrow{\mathbf{M}})$ la cuádrica $\mu$. Podemos denominar a $q$ unidad de intervalo.

No es difícil diseñar experimentos cinemáticos que permitan poner de acuerdo a otro observatorio $\mathscr{Q}_{\bar{\omega}}$ para que "ajuste» sus unidades de longitud y tiempo, de forma que den lugar a la misma unidad de intervalo $q$. El ajuste es único, y hace que la velocidad escalar de la luz desde $\mathscr{\ell}_{\bar{\omega}}$ tenga el valor $c$ :

El espacio afín $\mathbf{M}$, se transforma así, por medio de $q$, en una variedad de Lorent $z_{,}$con una orientación tiempo (en la que el vector $\omega_{1}$ apunta hacia el cono positivo). Este es el espacio afín de Minkowski, que constituye el-modelo 
geométrico clásico para el estudio de la cinemática relativista. El Capítulo V de [1] constituye una excelente referencia para este estudio.

\section{Bibliografía}

[1] O'NEIL, B.: Semi-Riemannian Geometry with applications to relativity. Academic Press. Inc., New York (1983).

[2] Einstein, A. The Meaning of relativity. Princenton University Press (1922).

[3] Tisseron, C.: Géométrie affine projective et euclidienne. Hermann, 1983.

[4] Berger, M.: Géométrie. Fernand Nathan, 1979.

Departamento de Geometría y Topología.

Facultad de Matemáticas.

Universidad Complutense de Madrid $\quad$ Recibido: 5 de abril de 1991 28040 Madrid. Spain 